\title{
Enhancing Change Capacity. Client-Consultant Collaboration and Emergent Change*
}

\author{
Kenneth W. Kerber ${ }^{* *}$, Anthony F. Buono ${ }^{* * *}$
}

\begin{abstract}
Companies, in every industry are increasingly challenged to build a capacity for change, both in response to and in anticipation of continuously changing competitive market and technological pressures.

Client-consultant collaboration can be prompted to broaden change management repertoire, creating a culture in which organizational members understand and support different approaches to change, ask and respond to questions that stimulate change and, in general, develop a culture of change.

Consultants, working and collaborating with the client, can prompt a broader appreciation of the range of appropriate change approaches and when turning a system 'loose' may be the most effective way to proceed. The goal is to work with clients to create organizations that are 'built to change'.
\end{abstract}

Keywords: Management Consulting; Consulting; Client-Consultant Relationship; Managing Organizational Change; Change Management; Globalization

\section{From 'Linear' to 'Complex' Change}

Companies in every industry are increasingly challenged to build a capacity for change, both in response to and in anticipation of continuously changing competitive, market and technological pressures. Given this need, significant attention has been devoted to conceptualizing and empirically testing a range of change management practices. Much of this effort, however, has focused on how to

\footnotetext{
* An earlier version of this article was presented at the Third International Conference on Management Consulting, Management Consulting Division, Academy of Management \& Copenhagen Business School, Copenhagen Denmark, May 31-June 2, 2007

** Kerber \& Associates, Chairman (kenkerber@ charter.net)

*** Professor of Management, Bentley College (abuono@bentley.edu)

Edited by: ISTEI - University of Milan-Bicocca

ISSN: 1593-0319

Kerber Kenneth W., Buono Anthony F., Enhancing Change Capacity. Client-Consultant Collaboration and Emergent Change, Symphonya. Emerging Issues in Management (symphonya.unimib.it), n. 1, 2007, pp. 81-96

http://dx.doi.org/10.4468/2007.1.10kerber.buono
} 
best implement specific organizational changes. While this emphasis has improved the ability of organizations to deal with the myriad ramifications of change, many of these models and prescriptions continue to fall short of the challenge (cf. Abrahamson, 2000; Beer, Eisenstat \& Spector, 1990; Kerber \& Buono, 2005). An underlying need is to develop the ability of organizations and their members to change not just once, but continuously, in response to ever shifting market conditions, customer demands and competitive pressures.

Many organizations are growing increasingly comfortable with planned change, especially in terms of their ability to react to external forces, define their preferred future state, and implement the subsequent 'plan' for achieving a well-defined end. In this context, however, change is largely viewed as linear and mechanistic, as a series of discrete and, at times, traumatic events that need to be controlled to enable organizations to achieve their goals. Given the onslaught of changes that a growing number of organizations now face, this carefully planned approach is quickly becoming inadequate because the ability to adapt to a rapidly changing environment also demands experimentation and improvisation, in essence continuous movement toward a largely unknown, emergent future state.

Beyond the abilities necessary to achieve planned change, emergent change requires a broad and deep organizational capacity for change that includes (1) the willingness and ability of change makers (change recipients, change agents/ implementers, change leaders/strategists) to assume responsibility for continuous changing, (2) an infrastructure that makes continuous changing possible (e.g., lavish communication, flexible systems and processes, responsive training and education); and (3) sufficient resources devoted to changing (e.g., mind share, time, people, money) (see Kerber \& Buono, 2005). Drawing on our consulting experience in this arena, the analysis focuses on the challenge of working with client organizations to stimulate emergent change. Emphasis is placed on the process of creating self-organizing systems and enhancing the client organization's change capacity.

\section{Managing Organizational Change}

From a conceptual vantage point, there are three interrelated approaches to organizational change that are present in organizations today: directed change, planned change and guided changing (Kerber \& Buono, 2005). Each of these approaches has certain advantages and disadvantages. Directed change is driven from the top of the organization, relies on authority and compliance, and focuses on coping with people's emotional reactions to change. Leaders create and announce the change and seek to persuade organizational members to accept it based on business necessity, logical arguments, and emotional appeals. Directed change reflects a quick, decisive approach to introducing change in an organization. Used inappropriately, however, managers and employees throughout the organization are forced to cope with the well-known and expected reactions of the recipients of the imposed change - denial, anger, bargaining, sadness, and loss (e.g., Kubler-Ross, 1969; Marks, 2003). 
Planned change, which has become an increasingly popular approach to change management, may arise from any level in the organization but ultimately is sponsored at the top. Change leaders and implementers seek involvement in and commitment to the change by making extensive use of specific actions, identified through research and experience, that mitigate the typical resistance and productivity losses associated with directed change (e.g., Beckhard \& Pritchard, 1992; Kotter, 1996; LaMarsh, 1995). Underlying most planned change efforts is the Lewinian three-stage process of unfreezing, changing, and refreezing: (a) unfreezing or releasing the organization from its current patterns, (b) transitioning the resulting, more malleable, organization from its current patterns to more adaptive ones, and then (c) refreezing the organization into a new set of patterns by weaving them into the fabric of the organization (Lewin, 1947, 1951; Weick, 2000; Weick \& Quinn, 1999).

Rather than simply creating and announcing the change, planned change provides a 'roadmap' that outlines a project management approach to the change process. It attempts to create the conditions for people to become more involved in the change process, identifying and encouraging key stakeholders to participate in both the form and implementation of the change. Yet, while planned change creates an important capability in today's organizations, used inappropriately it can still result in significant reductions in productivity, overwhelm organizational members with its complexity, alienate key stakeholders as a result of limited participation and true influence in the process, and constrain the ability of the organization to achieve its intended goals (cf. Abrahamson, 2000; Kerber, 2001). Moreover, the burden for initiating and sustaining the change is still placed directly on the change strategists, from identifying the need for change and creating a vision of desired outcomes to deciding which changes are ultimately feasible.

The planned change approach implicitly assumes that organizations experience inertia and that leaders must intentionally create change and consciously attempt to minimize resistance to that change. Yet, observations of organizations today suggest that it is increasingly common for change to arise from all levels in the organization, for people to make both small and large changes in their work based on trial and error and success and failure, and for changes initiated in one part of an organization to spread to other parts of the company. The reality is that such continuous changing is a natural part of organizational life (Wheatley, 1999).

Guided changing emerges from within the organization and people's commitment and contributions to the purpose of the organization. In the context of the type of over-lapping changes that are characteristic of today's hypercompetitive environment, this approach largely focuses on enhancing and extending the effects of the myriad changes that are already underway. It attempts to take full advantage of the expertise and creativity of organizational members, as organic changes emerge and evolve, reconfiguring existing practices and models, and testing new ideas and perspectives. Reflecting on Lewin's (1947, 1951) seminal contribution to planned change, guided changing follows a different three-stage process: freezing, rebalancing/ improvising, and unfreezing (Weick \& Quinn, 1999). Specifically, guided changing involves 'pausing' the action in an organization, at least figuratively, so that sequences, patterns, and interrelationships can be identified (freezing). Change agents can facilitate this process through vision casting and the 
creation of cultural maps that link different change efforts and initiatives. Based on a clearer understanding of what is happening in the organization, patterns can then be reassessed - re-shifting, rebalancing, and/or re-sequencing as necessary - to eliminate obstacles and blockages to these emergent changes (rebalancing/improvising). Instead of telling people what to do and why to do it (logic of replacement), the underlying key is to inspire organizational members so that they are drawn to and excited by the possibility of change (logic of attraction). At this point, the 'pause button' is figuratively released, unfreezing the action and resuming the learning and improvisation that characterize the guided changing process. Much of the ideal underlying guided changing is reflected in appreciative inquiry and its cycle of discovery, dream, design, and delivery (see Srivastava \& Cooperrider, 1990; Watkins \& Mohr, 2001).

Figure 1: The Guided Changing Spiral

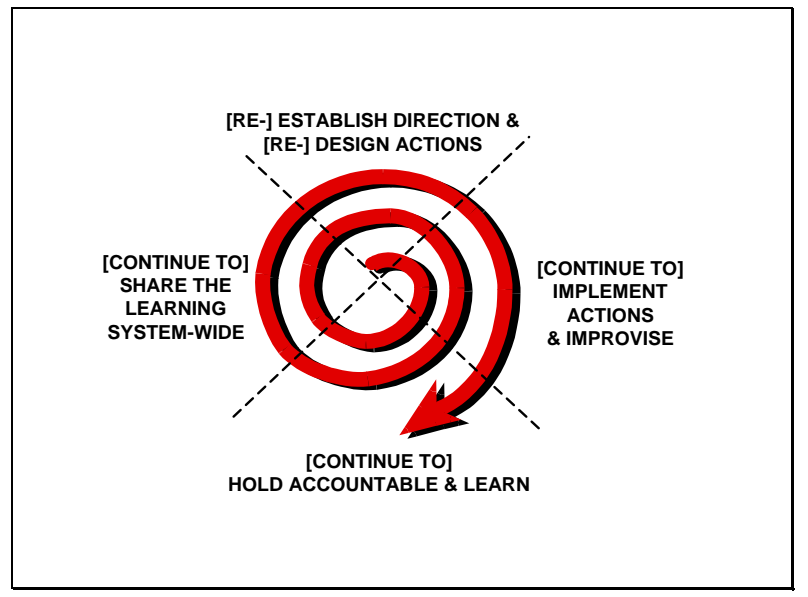

Source: Adapted from Kerber and Buono (2005)

As illustrated in Figure 1, guided changing is an iterative process of initial interpretation and design, implementation and improvisation, learning from the change effort, and then sharing that learning system-wide, leading to ongoing reinterpretation and redesign of the change. The resulting spiral of learning, innovation, and development contributes to both continuous improvement of existing change efforts as well as the ability to generate novel changes and solutions. Our experience suggests, however, that if used inappropriately, guided changing can contribute to organizational chaos, as continuous changes and transitions confuse and frustrate rather than enlighten and support organizational members and other key stakeholders. The feeling of being in 'permanent white water' (Vaill, 1989) and the need to constantly adapt and adjust can be a daunting experience. Many people ultimately want organizational change to end, rather than experience changing as a way of doing business that will, in essence, never end.

As each of these techniques has advantages and disadvantages, developing true change capacity entails the ability to move back and forth among these three change management approaches as required by the situation. Moving from directed change to planned change to guided changing, however, involves an underlying need for significant competence transfer from change strategists to change 
recipients, as organizational members become, in effect, the new change leaders (see Kerber \& Buono, 2005). The transition from planned change to guided changing, in particular, can pose a significant challenge for executives and organizational members, who are both accustomed to having the former lead change. While resistance is clearly related to instances where people feel change is being thrust upon them, a downside of carefully orchestrated planned change is that it might create an artificial sense of security among recipients that could counteract reflection (Werr, Stjernberg \& Docherty, 1997) and, as a result, suppress the type of learning and improvisation necessary for guided changing. At the same time, one of the greatest challenges to the implementation of guided changing may be the unwillingness of change strategists to let go of tight management control and embrace a messy, dynamic process that involves the entire organization (Kerber, 2001). It is imperative, therefore, that all organizational members - in essence the changemakers - become better informed about the advantages and limitations of each approach to change as well as the broader context for the change itself.

\section{Creating a Culture of Change}

Although many client organizations are faced with the need to stimulate emergent, guided changing, given their familiarity with directed and planned change approaches, it is typically most effective to begin by enhancing client understanding of how to successfully lead planned change in their specific context. Based on our experience, attempts to introduce guided changing concepts early on in the process are typically met with misunderstanding, skepticism, and/or resistance. Much like the idea of emergent change itself, it is more productive to have the motivation to seek out a different approach to change come from the organization and its members.

Starting with Planned Change. In consulting engagements/workshops we have conducted, we typically start with a series of questions that prompt participants to focus on their personal insights about the change process: (1) What is your most memorable experience with organizational change? Why is it so memorable? (2) Based on your experiences with organizational change, what is required to implement change successfully? (3) What specific change initiatives at XYZ Co. are you currently leading (or can influence)? What challenges do you face?

Drawing on the contributions of a number of thought leaders (e.g., Beckhard \& Pritchard, 1992; Beer \& Nohria, 2000; Kotter, 1996; LaMarsh, 1995), we then work with client organizations to develop a framework to conceptualize the process of managing planned change within the company: (1) identifying the change (including creating dissatisfaction with the status quo, establishing a clear vision for the change, developing a clear process for achieving that vision); (2) organizing the change project (creating a project plan and project team, ensuring technical expertise); (3) engaging the stakeholders (developing sponsorship, identifying innovators and early adopters, positioning skilled change agents, understanding the recipients of change); (4) implementing the change (managing the organization's history of change and cultural 'rules,' communicating the change vision and process, providing education/training as 
well as rewards/recognition); and (5) monitoring and sustaining the change (ensuring short-term wins, tracking metrics, problem-solving, collecting feedback, learning, sharing/disseminating the learning). As part of developing a better understanding of the planned change process, a powerful approach is to prompt client organizations to check regularly on the effectiveness of these key factors during the course of the planned change project. As an example, Appendix 1 contains a questionnaire designed to encourage conversation among the change implementers about these factors at critical stages during the life of the change project.

While this approach is effective in helping clients to more fully understand the dynamics surrounding changes that have a precisely defined beginning and end state, subsequent discussion typically reveals frustration with the limits of this approach. In prompting the reasons underlying their frustration, clients typically point to situations from their own experience that start with little more than a general direction, without a clearly defined end state, because the solution (or outcome) is generally not determined, established or exactly known. This realization lends itself to a discussion of the differing nature of organizational problems or challenges - in essence the distinction between what might be thought of as relatively 'tame' and 'wicked' problems (see Figure 2) (Conklin, 2005).

Figure 2: The Nature of Organizational Problems

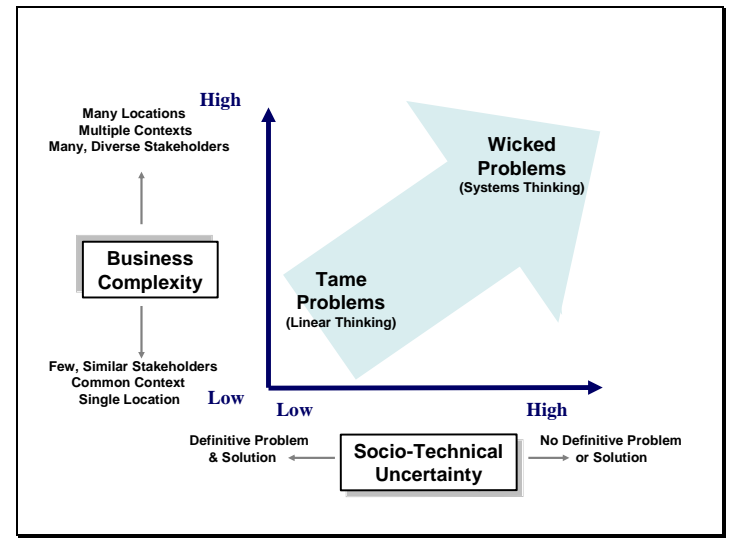

Source: Adapted from Conklin (2005)

Tame problems are those challenges that involve relatively few, similar stakeholders with a common context. The problem itself is clearly defined with a known solution. Wicked problems, in contrast, are those that demand a different approach and way of thinking. In these situations, the context itself can be very different (e.g., multiple contexts with many, diverse stakeholders) in which the problem is not clearly defined and the solution is also unclear. In essence, the initial focus on planned change opens clients to an iterative, rather than linear, approach to change that involves establishing an overall direction, taking action, assessing results, learning, sharing that learning, and adjusting the direction as they move toward and refine their goal(s). The desire to understand and learn how to guide emergent change when confronted with such wicked problems ultimately comes from the client organization. 
Developing a Diagnostic Orientation. As clients discuss the different nature of organizational problems, they also begin to question the limits of planned change. At this point, it is useful to prompt clients to think about the challenges and skills necessary to 'move forward' in dealing with 'wicked' as opposed to 'tame' problems. Within this context, the next step is to introduce them to the ways in which business complexity (i.e., the number of different components and extent of organizational differentiation) and socio-technical uncertainty (i.e., the extent to which the tasks involved are determined, established, or exactly known) also shape and influence change management dynamics, moving from directed and planned change when dealing with 'tame' problems toward guided changing when confronted with 'wicked' challenges. As illustrated in Figure 3, the primary driver of the shift from directed change to planned change is business complexity, while the shift to guided changing is driven by socio-technical uncertainty. Clients can begin to see the need for more of an iterative approach to change as they wrestle with problems and issues that are not clearly defined.

Figure 3 : Complexity, Uncertainty and Approaches to Change

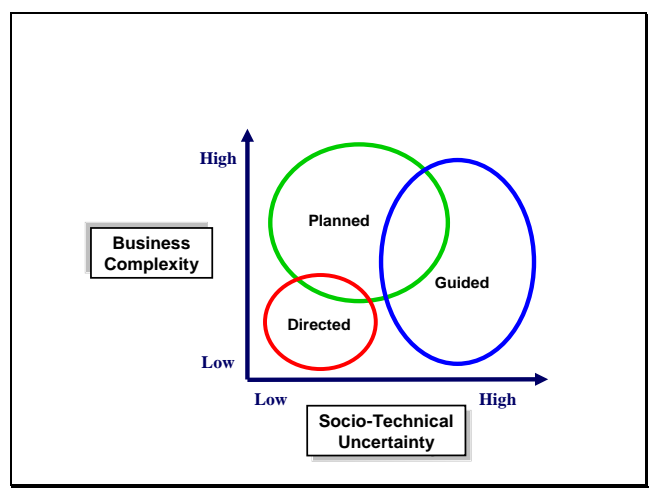

Source: Kerber \& Buono (2005)

Building Support for Guided Changing in a Large and Medium-Sized Corporation. Our recent experience with two client organizations - a multi-billion dollar information management technology company (IM Tech) and a medium-sized firm that engineers, installs and maintains state-of-the-art access control and security management systems (SMS Co) - shows how the limits of planned change can gradually become apparent in a complex, hyper-competitive, high change business environment.

- Strongly committed to leadership, management, and employee development, the director of IM Tech's corporate university asked us to design a workshop that would encourage leaders at the company to understand and apply a planned change approach when implementing significant organizational changes. After nearly a year of delivering workshops in the organization, a global sourcing initiative within the company's information technology organization provided an opportunity to consult with the $\mathrm{CIO}$ and his leadership team about how best to plan, announce, and implement a decision to offshore critical 
services to India. While the objective of this initiative was clear and well-defined (low socio-technical uncertainty), its implementation in a large, globally dispersed and culturally diverse organization was quite complex (high business complexity).

As reflected in the situational model of organizational change (see Figure 3), a planned change approach fit this moderately 'tame' situation.

Although not without its challenges, the implementation of the offshoring initiative was perceived to be sufficiently successful and well-received by the organization's managers, despite the obvious difficulty of offshoring desirable technical jobs, that leaders in other parts of the company took notice and requested customized workshops that would bring the planned change approach to their executives and managers. In particular, the customer service organization embraced a plan to educate its employees about thriving in a high change environment and its executives and managers about effectively leading planned change.

The customer service organization was faced with a wide range of organizational changes, all happening simultaneously including the introduction of new products and services, the integration of people and products from multiple acquired companies, major changes to internal processes and systems, offshoring, significant growth especially in developing countries, new strategies designed to have customers pay for valued services previously offered at no or low cost, and, as a result, new relationships with the company's marketing and sales organizations, not to mention the company's external customers. While some specific organizational changes, such as the implementation of new processes and systems, were well-suited to a planned change approach, the executives in customer service began to express dissatisfaction with the notion that planned change requires a clear description of the desired future state of the organization. During one executive overview of the planned change approach, for example, a participant described his dilemma with an analogy about completing a jig saw puzzle. Most of us are familiar with the experience of pouring 1,000 pieces of a complex jig saw puzzle on a table and then using the picture on the box cover to guide the assembly of the puzzle.

$\square$ As this executive noted, however, 'Working in customer service is like having someone pour an additional 1,000 puzzle pieces on the table, mixing them with the pieces already there, and then taking away the box cover. You have to put the puzzle together, and you need to start now, but you're not sure what you're building!' This analogy led to an impromptu discussion about the three approaches to change - with a focus on guided changing. The executives at the session embraced the notion of guided changing as describing their situation. In fact, during the discussion, one executive raised the question, 'So, when is each approach to change most appropriate?'

A similar dynamic took place in SMS Co. Following a series of training sessions (spread out over a four month period) in which the different approaches to change were discussed, the management team wrestled with how to best implement planned change within the firm. The idea of guided changing was seen as an 
intriguing but impractical approach to dealing with change in their organization. In a follow-up session that focused on reinforcing and applying the development tools discussed during the training sessions, the group began applying a planned change approach to honing and changing the way in which project bids were determined and integrated among the different areas of the company. As they were working through the process, it became increasingly clear that a planned change approach was falling short of what they needed to do, especially in terms of continually adapting to changing project specifications and customer expectations. As we were discussing the problem and the limitations of the current bidding process, one of the participants noted, '... hey, wait a minute. This seems like a guided changing challenge - like we talked about in the training sessions.' As others began to chime in, the group re-visited the guided changing spiral (Figure 1), using it to think through how the bidding process could be better integrated across all of the key areas of the company, with an emphasis on improvisation and sharing learnings throughout the organization.

In essence, starting with an understanding and application of planned change, where they felt the most comfortable, the executives and managers at IM Tech and SMS Co began to realize from their own experience that planned change, while necessary and effective in many situations, is not sufficient for all situations. As part of the intervention strategy with these two companies, we are now in the process of broadening their understanding of the alternative approaches to change, when each is appropriate, and how to implement each as effectively as possible. It appears that both client organizations are well on their way toward creating a culture of change.

Supporting Guided Changing in a Start-up Organization. Although many client organizations are initially leery about guided changing, this approach is already quite common on the Internet (e.g., open-source software development). As described in the two examples above, one can also expect to uncover pockets of guided changing within medium- to large-size organizations. Guided changing, however, may be received more openly and enthusiastically in small start-up organizations, especially those focused on experimentation and innovation (e.g., the life sciences).

As an example, the president of MedCo, an approximately 200person internal start-up within a large medical instruments company, approached us about preparing the start-up for anticipated explosive growth as a revolutionary product was being developed and brought to market. The president believed that creating a 'world class' organization and establishing a unique, supportive organizational culture were critical enablers to achieving the start-up's business objectives, especially during the expected period of fast growth. He also believed that his leadership team and the entire organization needed to own the process of creating such a world class organization and culture.

The challenge, however, was that neither the president nor his leadership team knew exactly what a 'world class' organization would look like in their unique 
environment. In fact, during our first meeting, the president said, 'I want to create a world class organization, and in all honesty, I'm not sure what that means. But I'll know it when I see it!' Similar to the puzzle pieces analogy, the goal was to start changing in the present but without a clearly defined vision of the future. Although the business complexity of MedCo was not particularly high, the uncertainty about the future state presented a 'wicked' problem, pointing to guiding changing as the most appropriate approach.

As we became involved with this internal start-up, we discovered that some steps in this direction had already been taken. The president had written and distributed several memos describing his preliminary ideas about the characteristics of a world class organization and company culture. The leadership team then brainstormed ideas about how world class organizations operate.

Based on focus groups with over 60 employees, actions had also been taken to improve company meetings, enhance overall internal communication, and develop the on-boarding (socialization) process for new employees. As a next step, we worked with the president and his human resources vice-president to design a one-day offsite for the senior leadership team that would establish a baseline for further action - education about guided changing, and a discussion about the aspects of 'world class' that currently needed focus, with time set aside for action planning.

In other words, the offsite was designed to define and initiate a long-term, iterative process for creating a world class organization and respective culture by building on the work that had already been done (e.g., the president's memos, the brainstorming session, the focus groups, improvement activities), and by taking the next step by re-defining and continuing to implement the next round of improvements (see Figure 4).

Figure 4 : Guided Changing at MedCo

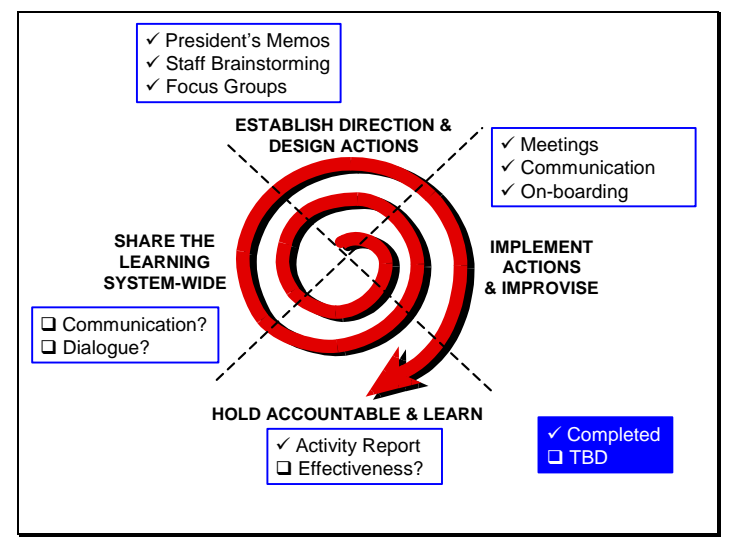

The underlying process was to establish and communicate an overall, if rather vague, direction for the company, take action to improve the organization, learn from that experience, share the learning with the entire organization, and take action again - an iterative process moving toward a clearer, emerging definition and realization of 
the desired world class organization and culture. As an analogy, while driving a car on a dark, moonless night it is possible to travel hundreds of miles safely and effectively only seeing as far ahead as your headlights allow. It may take years for this start-up organization to achieve 'world class' status, and they may never get there because the goal is a moving target. At the same time, concrete steps have begun and are likely to be continued, with the objective of moving the organization in the 'right' overall direction and adjusting the direction as it moves toward refining their ideal of creating a world class organization.

Figure 4 attempts to capture the activity at MedCo on the guiding changing spiral. It also points to an underlying challenge with guided changing - organizational members tend to uncomfortable in the 'Hold Accountable and Learn' and 'Share the Learning System-wide' steps in the process. It could be that these two steps are less action-oriented and more reflective than the first two. Given people's eagerness to jump into action, these reflective, learning, and communication activities may be reduced or eliminated - unless organizational members are prompted to undertake them. This is an area where consultants can play a critical role, especially since an emphasis on such learning and sharing is a crucial component of implementing guided changing.

\section{Thinking About Managing Change}

Directed change, planned change, and guided changing may be conceptualized along a continuum from highly constrained to highly unconstrained self-organization (see Figure 5). Self-organization is a process in which the internal organization of an open system increases in complexity without being tightly managed by outside sources (Olson \& Eoyang, 2001). The assumption here is that any group of people has the potential to self-organize, in other words, to become more organized and to generate new structures and patterns, that is, to change, based on the parameters of the situation and the dynamics of the group. Leaders constrain or unleash self organization by the way they design the parameters of the situation. Leaders may choose to constrain people so that they behave like a machine, or they may choose to unleash people so that they behave as a living system.

Figure 5: The Change Continuum

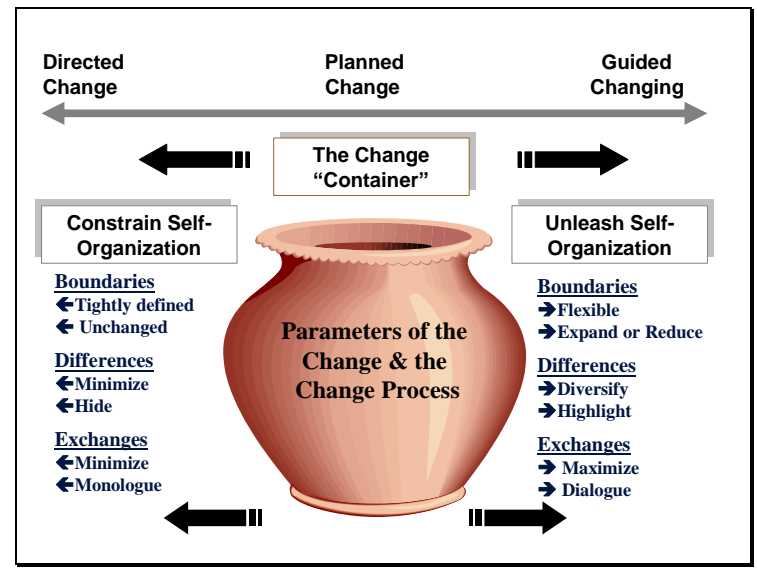


As illustrated in Figure 5, three primary factors influence the degree of selforganization in a system - the 'container' with its defining boundaries, the extent of differences within the container, and the types of exchanges that occur among the participants. If (1) the parameters of a particular change are tightly defined and unchanging, (2) the participants in the change process have few differences and those that do exist are hidden or suppressed, and (3) discussions about the change are minimized and communication about the change is one-directional from the leader, self-organization will be reduced or eliminated. In other words, directed change involves telling people what to do and how to do it, with little or no opportunity for input about or modification of the change goal or process. At the opposite extreme, as (1) the parameters of a particular change become increasingly flexible, (2) the participants in the change process are diverse and the differences are highlighted and celebrated, and (3) discussions about the change are maximized with a focus on true dialogue, self-organization is increased. In this instance, guided changing involves identifying an overall direction and then giving people the opportunity to modify both the change goal and the change process. Planned change falls in between these two extremes, for example, when people are involved in designing how to implement a relatively unchanging goal.

Figure 5 suggests that rather than three approaches to change, there are in fact an infinite number of change approaches along a continuum from tightly constrained to highly unconstrained. Our experience suggests that once organizational members begin to understand the various approaches to change, they are quite capable of determining the appropriate change strategy - if given the opportunity. As an example, Figure 6 is a snapshot of a flip chart from a recent intervention at IM Tech, with those in the workshop indicating which approach to change they thought was most appropriate for the challenge(s) they were facing. By assessing the relative business complexity and socio-technical uncertainty associated with their situation, the flip chart suggests that they were quite capable of determining where they should be operating along the change continuum. Discussion also focused on working at the boundaries, as the types of change strategies blur together in practice. As one moves from directed change, to planned change, to guided changing, leaders must give up control based on rules, procedures and tight supervision and substitute self-organization based on overall direction, principles and values.

As captured in Figures 3 and 5, the appropriate degree of self-organization depends on the nature of business situation and change challenge. Too frequently in today's organizations, people are overly constrained based on the leaders' need for control and predictability in contrast with what is required by the circumstances. In the appropriate situation, unleashing self-organization can have very positive benefits, including engagement, creativity, innovation, and commitment to change. The greatest challenge to building support for guided changing may be the unwillingness of leaders to let go of tight management control and embrace a messy, dynamic process that involves the entire organization. In situations requiring creativity and innovation, leaders must be more willing to trust the intelligence throughout the system, not just a relatively small number of experts or people at the top. 
Figure 6: Conceptualizing Change Strategies at IM Tech

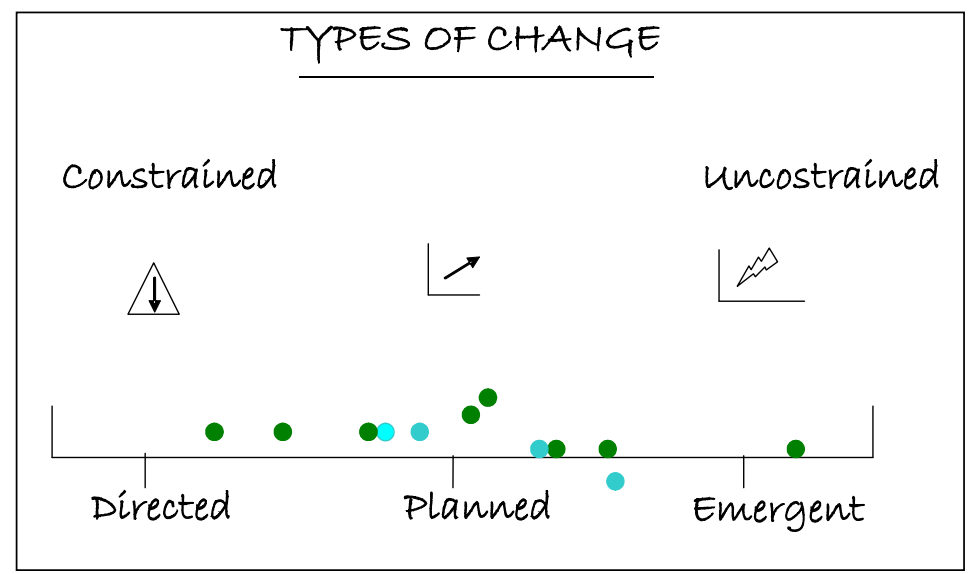

\section{Conclusion}

Client organizations can be prompted to broaden their change management repertoire, creating a culture in which organizational members understand and support different approaches to change, ask and respond to questions that stimulate change (e.g., the 'architecture of possibility,' see Finlayson, 2001), create a supportive change infrastructure, and, in general, develop a high capacity for change. The initial impetus for moving from directed to planned change, which is driven by business complexity, can be managed as part of a traditional training and development approach, broadening the way in which organizational members view successful change. From a consultancy perspective, however, the impetus for moving from planned change to guided changing, which is driven by sociotechnical uncertainty, must come from the client organization. As discussed in the paper, clients are often leery of the idea of unconstrained systems, seeing them as more ideal than realistic, until they experience the limitations of planned change. This dynamic, of course, may change over time as organizations become more familiar with these different approaches to change. It is in these instances that consultants, working and collaborating with the client, can prompt a broader appreciation of the range of appropriate change approaches and when turning a system 'loose' may be the most effective way to proceed. The goal is to work with clients to create organizations that are 'built to change' (Tichy, 1998), not just once, but on an ongoing basis in response to and in anticipation of a continually changing environment.

\section{Bibliography}

Abrahamson E., Change without Pain, Harvard Business Review, vol. 78, n. 4, 2000, pp. 75-79.

Beckhard R., Pritchard W., Changing the Essence, Jossey-Bass, San Francisco, 1992.

Beer M., Eisenstat R., Spector, B., What Change Programs Don't Produce Change, Harvard Business Review, vol. 68, n. 6, 1990, pp. 158-166. 
Beer M., Nohria, N., Cracking the Code of Change, Harvard Business Review, vol. 78, n. 3, 2000, pp. 133-141.

Conklin J., Wicked Problems and Social Complexity: Dialogue Mapping - Building Shared Understanding of Wicked Problems, Wiley, Hoboken, NJ, 2005.

Finlayson A., Questions that Work, American Management Association, New York, 2001.

Kerber K. W., Change in Human Systems: from Planned Change to Guided Changing, A. F. Buono (ed.), Current Trends In Management Consulting, Information Age Publishing, Greenwich, CT 2001, pp. 145-169.

Kerber K. W., Buono, A. F., Rethinking Organizational Change: Reframing the Challenge of Change Management, Organization Development Journal, vol. 23, n. 3, 2005, pp. 23-38.

Kotter J. P., Leading Change, Harvard Business School Press, Boston, MA, 1996.

Kubler-Ross E., On Death and Dying, Macmillan, New York, 1969.

LaMarsh J., Changing the Way We Change, Addison-Wesley, Reading, MA, 1995.

Lewin K.,. Field Theory in Social Science, Harper \& Row, New York, 1951.

Lewin K., Frontiers in Group Dynamics, Human Relations, vol. 1, n. 1, 1947, pp. 5-47. http://dx.doi.org/10.1177/001872674700100103

Marks M. L., Charging Back Up the Hill: Workplace Recovery After Mergers, Acquisitions, and Downsizings, Jossey-Bass, San Francisco, 2003.

Olson E.E., Eoyang, G.H., Facilitating Organization Change: Lessons from Complexity Science, Jossey-Bass/Pfeiffer, San Francisco, 2001.

Srivastava S., Cooperrider, D., Appreciative Inquiry and Leadership: the Power of Positive thought and Action in Organizations, Jossey-Bass, San Francisco, 1990.

Tichy N., The Leadership Engine, Pritchett LP, Dallas, TX, 1998.

Vaill P., Managing as a Performing Art, Jossey-Bass, San Francisco, 1989.

Watkins J.M., Mohr, B.J., Appreciative Inquiry: Change at the Speed of Imagination, Jossey-Bass, San Francisco, 2001.

Weick K. E., Emergent Change as a Universal in Organizations, M. Beer, N. Nohria (eds.), Breaking the Code of Change, Harvard Business School Press, Boston, 2000, pp. 223-241.

Weick K.E., Quinn R.E., Organizational Change And Development, J. T. Spence, J. M. Darley, D. J. Foss (Eds.), Annual Review of Psychology, Annual Reviews, Palo Alto, CA, 1999, pp. 361-386. http://dx.doi.org/10.1146/annurev.psych.50.1.361

Werr A., Stjernberg T., Docherty P., The Functions of Methods of Change in Management Consulting, Journal of Organizational Change Management, vol. 10, n. 4, 1997, pp. 288-307. http://dx.doi.org/10.1108/09534819710175839

Wheatley M., Leadership \& the New Science, Berrett-Koehler, San Francisco, 1999. 


\section{Appendix 1: Leading Organizational Change Questionnaire}

With reference to the $X W Y$ project, evaluate: [A] the importance of each element of successful change, listed below, to achieve the goals of the project, and [B] the effectiveness of the efforts thus far to use each change element to achieve the goals of the project.

Rate each item twice, once for importance (IMP) and once for effectiveness (EFF), with reference to the $X W Z$ project. Use the 0 to 10 scale as follows:

Unimportant IMPORTANCE Very Important

$\begin{array}{lllllllllll}0 & 1 & 2 & 3 & 4 & 5 & 6 & 7 & 8 & 9 & 10\end{array}$

Poor EFFECTIVENESS Excellent

1. Dissatisfaction with status quo (among the people who must change):

IMP $\mathrm{EFF}$

2. Clear vision for change (among the people who must change):

IMP EFF

3. Clear process for achieving the vision (among the people who must change):

IMP $\mathrm{EFF}$

4. Project plan (i.e., scope, milestones, schedule, and resources):

IMP $\mathrm{EFF}$

5. Project team:

IMP EFF

6. Technology and technical expertise to accomplish project milestones:

IMP EFF

7. Sponsorship:

IMP EFF

8. Perceived costs of change (among the people who must change):

IMP EFF

9. Innovators and early adopters:

IMP EFF

10. Skilled change agents:

IMP EFF 
11. The people who much change:

IMP EFF

12. History of change:

IMP EFF

13. Cultural 'rules':

IMP $\mathrm{EFF}$

14. Communication of the change vision and process (to all stakeholders affected by the change):

IMP EFF

15. Education/training (of all stakeholders affected by the change):

IMP EFF

16. Rewards/recognition (among all stakeholders affected the change):

IMP $\mathrm{EFF}$

17. Short-term wins:

IMP $\mathrm{EFF}$

18. Metrics:

IMP EFF

19. Problem-solving:

IMP EFF

20. Quality of feedback:

IMP EFF

21. Learning and sharing the learning:

IMP EFF 